\title{
Experimental and theoretical photochemistry: application to the cometary environment and Titan's atmosphere
}

\author{
M.-C. Gazeau*, H. Cottin, V. Vuitton, N. Smith, F. Raulin \\ L.I.S.A. (Laboratoire Interuniversitaire des Systèmes Atmosphériques), UMR 7583, CNRS, Universités Paris VII et XII, 61, Avenue du Général de \\ Gaulle, 94010 Creteil, cedex, France
}

Received 23 July 1999; accepted 11 October 1999

\begin{abstract}
Coupling the data obtained from laboratory experiments, theoretical modelling and observation is the way to reach a better understanding of the organic chemistry involved in a planetary or a cometary atmosphere. We discuss briefly here the different interactions between experimental studies and observations and develop the contribution of laboratory experiments to models in the case of both Titan and cometary investigations.

In particular, we point out the lack of fundamental data such as kinetic rate constants and quantum yields necessary to build theoretical models. We present a photochemical experiment dedicated to provide such parameters in order to support the theoretical modelling of interactions between the cometary nucleus and the coma.

We discuss the limitations of simulation experiments with respect to extracting useful data for modelling. We propose that the comparison of simpler, well-constrained laboratory experiments and theoretical models of these systems can lead to a critical examination of the accuracy of the model's chemical schemes. We illustrate this for the case of Titan.

These studies have direct implications for the preparation and the analysis of results from planetary space missions. (C) 2000 Elsevier Science Ltd. All rights reserved.

Keywords: Photochemistry; Laboratory experiments; Experimental simulations; Titan; Comets; Photodegradation; Polyoxymethylene; Planetary atmosphere; Cometary environment
\end{abstract}

\section{Introduction}

Planetary atmospheres and cometary environments can be studied by a range of complementary techniques: observational programs, theoretical modelling and laboratory experiments (Bruston and Raulin, 1995). Although each of these three approaches can individually yield useful information, our understanding of atmospheric systems can often be further improved by coupling these methods.

Analysis of observational data can yield information about many aspects of a planetary atmosphere, such as its chemical composition, or the deposition of

\footnotetext{
* Corresponding author. Fax: + 33-1-4517-1564.

E-mail address: gazeau@lisa.univ-paris12.fr (M.C. Gazeau).
}

energy and the resulting temperature profile. Such data can be obtained by remote sensing of the atmosphere by instruments based on, or in orbit around Earth, or for the case of other planets, space missions that fly by or orbit the planet in question. This approach can measure the relative abundances of the atmospheric constituents over a significant period of time, monitoring any variation which may occur. However, analysis of these observational data requires knowledge of the spectroscopic characteristics (frequencies, absolute band or line intensities) and their temperature dependence, for all atmospheric constituents. Unfortunately, there is often a lack of reliable laboratory measurements of these parameters.

There is a second means to observe an atmosphere, by performing in situ measurements using a descent probe (Raulin et al., 1996). This technique allows the 
measurement of the exact composition, temperature, pressure and wind profiles of a given planetary atmosphere, but is limited to only one trajectory in latitudelongitude-altitude-time coordinates. However, this kind of mission is very difficult to realize, hence few have been carried out to date.

The scarcity of observations implies a strong need for theoretical modelling. In order to calculate the composition of an atmosphere, its evolution and its spatial variation we need to develop photochemical models. These models contain descriptions of both physical and chemical phenomena. Therefore, a study of the accuracy of either the chemical or physical parts of the photochemical model cannot be effectively carried out by comparing its results with observations. We need to recognize the presence of two major sources of error in the chemical scheme of such a model in order to refine the theoretical descriptions of these processes (Smith, 1999). First, there are uncertainties in laboratory determinations of the values of the kinetic parameters used in the model (absorption cross sections, quantum yields and rate constants). Second, it is possible that critical reaction pathways are missing from the model, which can lead to systematic errors in the calculation.

Laboratory experiments can be used to reduce these two sources of uncertainty. Firstly, they can be used to determine missing kinetic data or to determine these parameters with greater precision over a range of temperatures and pressures. Secondly, they can also be performed to simulate the complex evolution of the studied environment, by submitting a given gas mixture to energy deposition under controlled conditions (Sagan et al., 1984). These simulations provide very useful information on the possible presence of minor compounds in planetary and cometary environments, and can highlight weaknesses in photochemical models, for example, by showing the presence of a compound that has not been considered by the model. In this case, the model needs to include novel pathways for the formation of these compounds. However, simulation experiments cannot easily be used to examine the accuracy of photochemical models, as the simulated system is highly complex, and it remains difficult to determine the dominant processes that occur. Nevertheless, by reducing the complexity of the system, it is possible to test a restricted part of the chemical scheme used in photochemical models. For example, following the photochemical evolution of a simple gas mixture can provide quantitative data which can be directly used to test the accuracy of the chemical scheme. In this way, we can test the comprehensiveness of the model.

Unfortunately, there are unavoidable limitations to the analysis of the results of all laboratory experiments due to the unknown contribution from wall effects. As models usually do not take into account these heterogeneous processes, discrepancies between model and experiment are not necessarily indicative of errors in the model's description of gas phase chemistry. However, from our point of view, comparison between theory and experiment remains the only way to reach a better understanding of these chemical systems, and a more accurate chemical scheme can be developed through coupling these approaches. Obviously, it is therefore essential to eliminate any wall effects from the experiment, or at least to study these effects in order to understand and quantify them.

In this paper, we present two experimental programs developed in our laboratory:

- The first one, named S.E.M.A.Ph.Or.E. COMETAIRE, consists of performing specific experiments devoted to the determination of fundamental parameters necessary to feed models of the cometary environment.

- The second one, named I.M.I.T.A.T.E.S., couples simple and well-constrained photochemical experiments with theoretical studies in order to improve the restricted part of the chemical scheme used in photochemical models of Titan's atmosphere.

\section{Cometary investigations}

\subsection{Review of the previous works}

Composition of the cometary environment has been investigated through traditional spectroscopic techniques from the UV-visible to the submillimetric and millimetric ranges. Thanks to both recent Earth flybys by comets Hyakutake and Hale-Bopp, many groundbased observations have been done. Until now, observations led to the detection of more than 20 stable small molecules in the coma of the comets (mainly $\mathrm{H}_{2} \mathrm{O}, \mathrm{CO}, \mathrm{CO}_{2}, \mathrm{CH}_{3} \mathrm{OH}$ ) (Crovisier, 1998).

Concerning numerical simulation, studies are not as advanced as they are for Titan's atmosphere. Indeed, it is very ambitious to link the four main aspects of the question: physics of the nucleus (mainly radiative transfer, differentiation into the nucleus, crystallisation of water ice, sublimation of compounds, ejection of grains), physics of the coma (ejection from nucleus by jets, transport in the coma, interaction with solar wind, plasma and dust tail formation), chemistry of the nucleus (reactions between the main compounds under thermal, UV or galactic cosmic rays processing) and the chemistry of the coma (photodissociation of molecules, reactions between them). The four aspects are studied separately but we have to keep in mind that they are tightly interconnected. For example, 
Irvine et al. (1998) and Rodgers and Charnley (1998) have shown that HNC in comet Hale-Bopp was not directly produced by the nucleus but in the gaseous phase by the chemistry of HCN. Recently, Crifo et al. (1999) have shown that day-to-night asymmetries in the CO molecule velocity may arise even with a uniform production all around the nucleus, and be due to the surface temperature distribution.

Experimental simulations have extensively been undertaken on ice mixtures, whether to understand trapping and release of gases in ices (Notesco and BarNun, 1996, 1997; Notesco et al., 1997) or to infer cometary chemical composition by irradiating ices with composition relevant to interstellar medium at $10 \mathrm{~K}$. The resulting sample, supposed to mimic the interstellar or cometary ice, is usually analysed by different methods.

Three main energy sources are used:

- heat (Schutte et al., 1993);

- UV lamps, and specifically hydrogen discharge lamp (Briggs et al., 1992; Gerakines et al., 1996; Bernstein et al., 1995);

- high energetic particules (1-100 keV): $\mathrm{H}, \mathrm{He}$ ions and heavier nuclei generated by a Van de Graff generator (Strazzulla et al., 1991; Kobayashi et al., 1995; Moore and Hudson, 1998).

The whole set of experimental simulations on ice analogues have been reviewed in Cottin et al. (1999) and leads to the detection of more than 50 compounds from simple molecules such as $\mathrm{CO}, \mathrm{CO}_{2}, \mathrm{H}_{2} \mathrm{CO}$ and $\mathrm{CH}_{4}$ to heavier ones belonging to almost each kind of the main chemical families.

\subsection{A new step: S.E.M.A.Ph.Or.E. COMETAIRE ( $a$ french acronym: Simulation Expérimentale et Modélisation Appliquées aux Phénomènes Organiques dans l'Environnement COMETAIRE)}

Starting from the molecular abundance of compounds in the coma determined from observations, it is rather difficult, even impossible, to infer the molecular composition of the cometary nucleus. We have seen that previous investigations on a cometary environment, using laboratory simulation experiments, have led to extrapolate to some extent the composition of the nucleus. But the resulting information is often qualitative and few quantitative data have been obtained so far. For example, from the Schutte experiment (Schutte et al., 1993) on ice analogs containing $\left(\mathrm{H}_{2} \mathrm{O}: \mathrm{CH}_{3} \mathrm{OH}: \mathrm{HCHO}: \mathrm{CO}: \mathrm{NH}_{3} ; \quad\right.$ 100:50:4:10:1), one could expect polyoxymethylene as well as other high molecular weight compounds to be present in the nucleus. The problem is that such species have not yet been detected. This is the reason why the presence of these molecules in the nucleus of comets is still an open question. In order to bring to a close this question, we proposed at L.I.S.A., a new experimental approach, named S.E.M.A.Ph.Or.R.E. COMETAIRE, (Cottin et al., 1999) consisting of irradiating high molecular weight compounds supposed to be present in the nucleus. The quantitative analysis of the molecular composition of the resulting gaseous phase gives the "own signature" of this molecule. In other words, the idea of this simple experiment is to determine what is the contribution in the gaseous phase of high molecular weight solid present in the nucleus.

We have also pointed out the need for quantitative data necessary for the build up of models. The S.E.M.A.Ph.Or.R.E. COMETAIRE program belongs to the first kind of laboratory experiments described in a previous section of this article as it provides fundamental parameters necessary to build accurate models on the evolution of the cometary environment. We are studying the transformation of high molecular weight compounds, suspected to be present in the nucleus, into photodegradation products. Abundance, rate and quantum yields of formation of the dominant products can be derived from the performed experiments. Retrieving such data would be of prime importance to analyse and interpret the results of previous observations as well as future cometary missions.

The scientific problems which are dealt with by S.E.M.A.Ph.Or.E. COMETAIRE are:

- the degradation of high molecular weight organics likely to be present in the nucleus when the comet approaches the inner solar system and is then reheated and submitted to UV photons from the Sun;

- the correlation between the nucleus and the coma's chemical composition;

- the understanding of the origin of the observed extended sources (i.e. compounds which are not directly emitted from the cometary nucleus, but certainly from photodegradation of larger molecules in the coma).

The first application of the experiment is the study of the photodegradation of polyoxymethylene (POM) under UV irradiation. We note that POM is not necessary present in the nucleus but POM seems to be the best candidate as parent molecule for the formaldehyde extended source (Meier et al., 1993). Furthermore, POM has been proposed as an interpretation of the heavy ion mass spectra obtained in the inner coma of comet Halley with the PICCA mass spectrometer on GIOTTO spacecraft (Huebner, 1987). Additionally, Möller and Jackson (1990) have performed mass spectrometry experiments on POM and have concluded that the PICCA spectrum is consistent with the hypothesis that the formaldehyde polymer is present in comets. Nevertheless, Mitchell et al. (1992) ruled out 
this hypothesis arguing that the pattern shown in the PICCA spectrum could be due to any CHON mixture and thus do not imply the presence of a polymer as POM. From our point of view, an important clue is that POM has been detected after thermal processing of ice analog containing formaldehyde (Schutte et al., 1993) and after UV irradiation of $\left(\mathrm{H}_{2} \mathrm{O}: \mathrm{CH}_{3} \mathrm{OH}\right.$ :$\mathrm{CO}: \mathrm{NH}_{3} ; 100: 50: 10: 10$ ) ice mixture (Bernstein et al., 1995).

\section{Titan's investigations}

\subsection{Review of the previous works}

Discovered in 1655 by Huygens, Titan, the largest satellite of Saturn, became an object of great interest after the suggestion by Comas Solà in 1908 (Solà, 1908) that it may have an atmosphere. The latter was characterized for the first time in 1944 by Kuiper (Kuiper, 1944): spectroscopic measurements from the Earth in the visible range have shown the spectral signature of methane. Since that time, many observations have been performed from the ground and other hydrocarbons have been quantitatively detected. Voyager spacecraft, in the early 1980s, have drastically improved our vision of the quasi-planet. The radio-occultation and the IR and UV spectrometers (IRIS and UVS experiments, respectively) aboard Voyager have allowed the determination of its vertical pressure/temperature profile, the main chemical composition $\left(\mathrm{N}_{2}\right.$ and $\left.\mathrm{CH}_{4}\right)$ of its atmosphere and to identify several trace atmospheric species. After Voyager, observations in the millimeter range or infrared measurements from ISO (Infrared Space Observatory) have provided complementary data on the chemical composition of Titan's atmosphere (see for example, Hidayat et al., 1997; Bézard et al., 1993; Cousténis et al., 1997).

The presence of a wide variety of minor species and hazes probably made of organic compounds suggest that an active and complex photochemistry takes place in Titan's atmosphere. Four one-dimensional models have been developed up to now (Strobel, 1974; Yung et al., 1984; Toublanc et al., 1995; Lara et al., 1996) to simulate the chemical evolution of Titan's atmosphere. These models were principally dedicated to test some hypothesis concerning the physical parameters involved in the determination of the chemical composition of the atmosphere. The obtained theoretical vertical mixing ratio profiles have been compared with observations with unequal success depending on the studied compound. Additionally, a recent work (Smith, 1999) has demonstrated that uncertainties in the rate constants used in the models can introduce very large error bars in the distribution of the atmos- pheric compounds. Once again, the crucial need of laboratory data is obvious.

In parallel, many laboratory experiments have been carried out in order to mimic Titan's organic chemistry. With the goal to simulate Titan's atmosphere, $\mathrm{N}_{2} / \mathrm{CH}_{4}$ gas mixture was submitted to irradiation with different energy sources (Thompson et al., 1991; McDonald et al., 1994, de Vanssay et al., 1995; Coll et al., 1998, and references therein). However, these simulation experiments were subject to criticism as they were performed with non representative pressure and temperature conditions, non representative energy sources and with the presence of wall effects. The more recent simulation experiments have been improved, using a silent discharge and flow system, within the correct range of temperature and pressure, carefully avoiding any contamination (Coll et al., 1999). The obtained data show a very good agreement with the observational data, demonstrating for the first time the formation of all the organic species already detected in Titan's atmosphere. Furthermore, in these experiments solid products are also formed. They are considered as chemical analogues of Titan's aerosols. Characterisation of these laboratory analogues by pyrolysis, elemental analysis and establishment of the optical properties is of prime importance. For example, observation of the satellite by remote sensing techniques needs to be treated by models, in which the optical properties and structure of these atmospheric particules have to be introduced.

\subsection{Titan's photochemical program: IMITATES \\ (Irradiation of Mixture of Interest for Titan's Atmosphere: Theoretical and Experimental Studies)}

Large discrepancies have been found between theoretical and observational data concerning the vertical mixing ratio profiles of some compounds in Titan's atmosphere. It must be pointed out that the photochemical models have been built with physical and chemical considerations. Thus, errors in the description of the physical processes as well as of the chemical mechanisms involved in this atmosphere can be invoked to explain the differences between theory and observation. The aim of our laboratory program is to better understand the photochemistry of Titan. As we said previously, a way to reach this objective is to test the chemical module (or part of this module) used in photochemical models. Then, the performed studies consist in comparing the experimental data obtained from our laboratory experiments carried out in perfectly known physical conditions with the theoretical data obtained with a $0-\mathrm{D}$ model built with reaction pathway parameters (rate coefficients, branching ratios, photolysis frequencies, etc) adapted to the considered experimental conditions. 
We are currently working on a simplified system allowing to test part of the global chemical module used in the photochemical models: the catalytic scheme of dissociation of methane, via the photolysis of acetylene, that is supposed to occur in Titan's stratosphere (Smith et al., 1999). Experimental irradiations of $\mathrm{CH}_{4} /$ $\mathrm{C}_{2} \mathrm{H}_{2}(1000 / 1)$ gas mixtures, at $1849 \AA$, have been performed. Three comprehensive chemical schemes (Lara et al., 1996; Toublanc et al., 1995; Yung et al., 1984) have been adapted to model the experiments. The experiments have already confirmed that dissociation of methane can be catalysed by the photolysis of acetylene at $1849 \AA$. However, we have found the measured quantities of resulting products to differ drastically from the predictions of the theoretical models and we explain this discrepancy through heterogeneous processes occurring on the walls of the reactor not taken into account in the models. Additionally, the dependence of the quantity of products as a function of time has been studied by conducting a series of experiments carried out independently. Consequently, due to nonreproducibility of the flux of the lamp between experiments, the results have been obtained with large error bars. Thus, a new program of experiments has been developed in order to avoid both limitations.

\section{Experimental}

Chemical analysis of the gaseous mixture resulting from the irradiation of the heavy organic molecules (cometary experiments) or $\mathrm{CH}_{4} / \mathrm{C}_{2} \mathrm{H}_{2}$ gas mixture with acetylene in catalytic amount (Titan's experiments) is carried out using a $1 \mathrm{~m}^{3}$ atmospheric simulation chamber (ASC), usually devoted to the study of tropospheric chemistry of the Earth (Fig. 1).

In the case of S.E.M.A.Ph.Or.R.E. COMETAIRE, the studied high molecular weight compound is simply deposited at the bottom of a reactor and irradiated with UV photons $(\lambda=147 \mathrm{~nm})$ delivered by a Xenon lamp. This experimental setup has already been described (Cottin et al., 1999). The reactor, made of pyrex, has been slightly modified in order to permit the thermal regulation of the sample (Fig. 2). This improvement was necessary to discriminate between thermal and irradiation effects. As the reactor is directly connected to the ASC, the gaseous photodegradation products simply diffuse from the reactor to the chamber (used then only as a very sensitive analytical technique) where they are continuously analysed during irradiation. In order to avoid desorption of molecules previously adsorbed on the walls of the atmospheric simulation chamber, one has to work in an

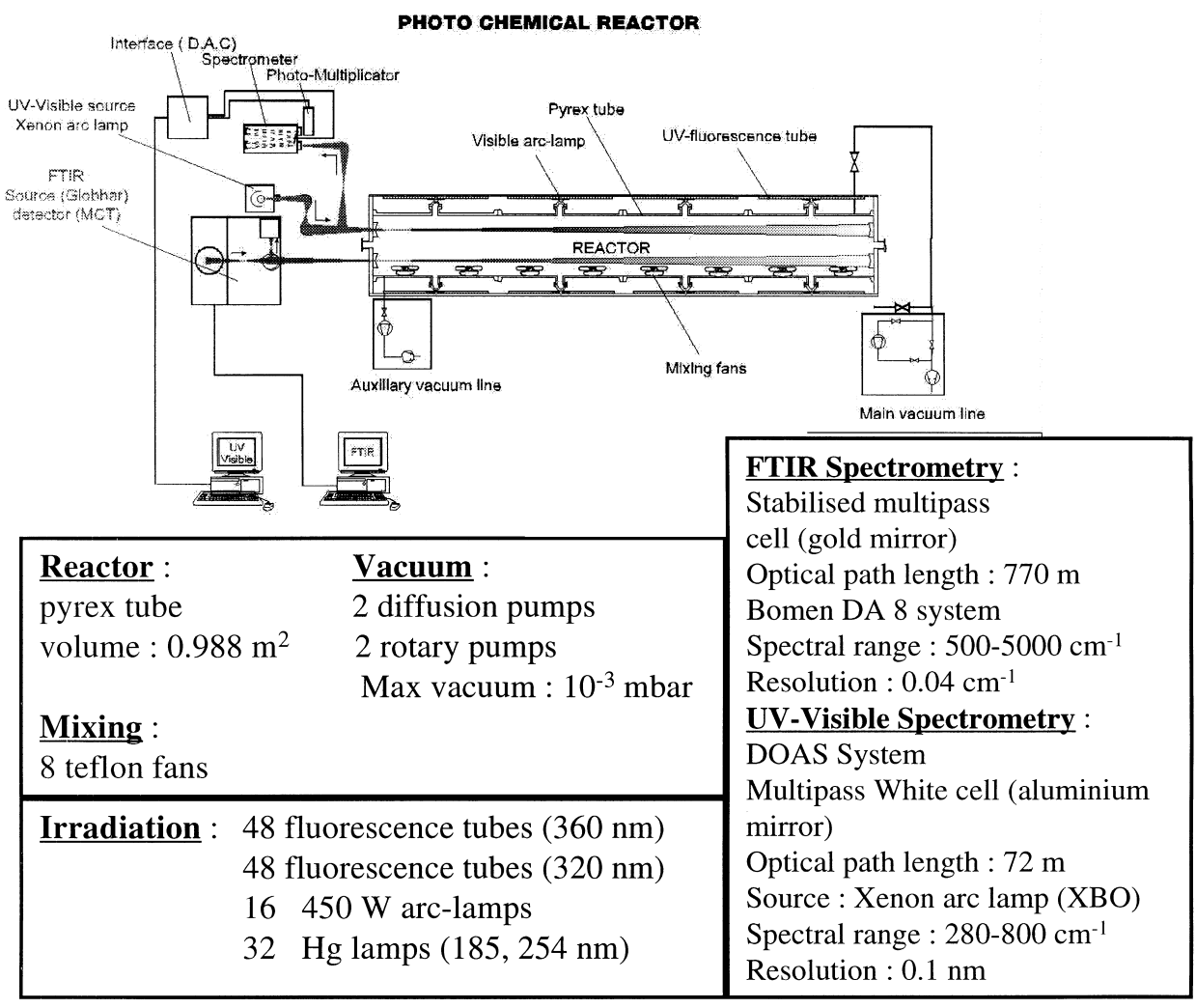

Fig. 1. Atmospheric Simulation Chamber at L.I.S.A. 
inert atmosphere of $2 \mathrm{mb}$ of $\mathrm{He}$ (Type C, Aga, France).

In the Titan experiments, the chamber is used both as a reactor and as an analysis system. As the $\mathrm{CH}_{4}$ / $\mathrm{C}_{2} \mathrm{H}_{2}$ gas mixture is directly irradiated in the chamber by UV photons delivered by mercury lamps, its evolution can be followed continuously during the whole irradiation time, which is a great improvement in comparison with our previous experiments. Moreover, this method allows to minimise wall effects which, as we have seen, are a serious limitation when comparing chemical models to laboratory experiments.

In both cases, the simulation chamber is used as a high performance spectrometer as it is equipped with two spectrometers: a long optical path UV-visible spectrometer (optical path length: $72 \mathrm{~m}$, range: 250 $650 \mathrm{~nm}$, maximum resolution: $0.05 \mathrm{~nm}$ ) and very long path FTIR spectrometer (optical path length: $672 \mathrm{~m}$, range: $600-4000 \mathrm{~cm}^{-1}$, resolution: from 64 to $0.003 \mathrm{~cm}^{-1}$ ) (Doussin et al., 1997). One can notice that the analysis can also be performed by mass spectrometry (MS) or gas chromatography coupled with mass spectrometry (GC-MS). In the cometary experiments and in the case of GC-MS analysis, preconcentration of the resulting gaseous phase with a cold finger is needed. In the Titan experiments, sampling of the gas mixture with a syringe allows its analysis after injection in the dedicated MS or GC-MS apparatus.

Concerning FTIR analysis, the identification of the different compounds is based on reference spectra from the EPA library (Daugherty et al., 1994, 1995). Calibration curves have been performed for all the detected species in order to allow their quantification. As an example, we present in Fig. 3 the infrared spectrum obtained for a 4-h irradiation of POM; the exper-

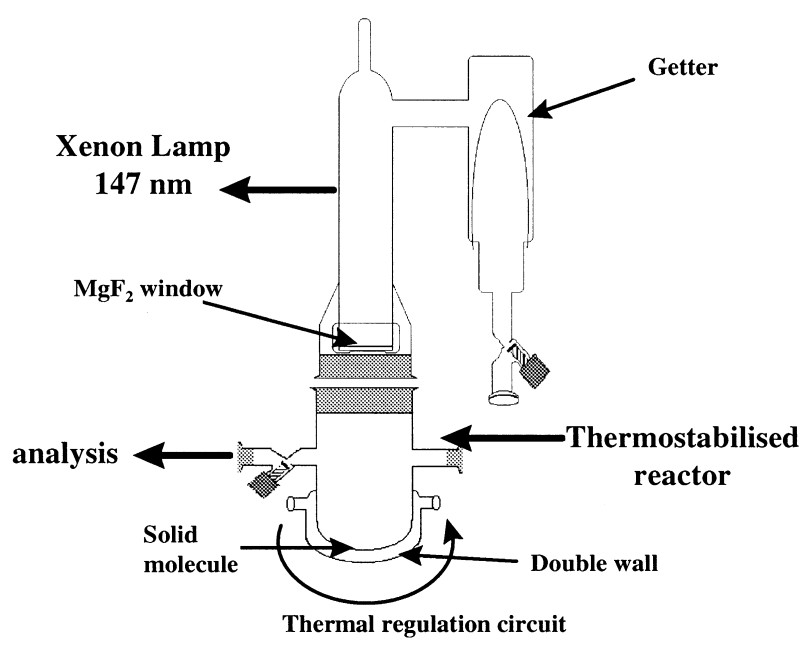

Fig. 2. Schematic view of the photochemical reactor used for the irradiation of solid materials. iment is performed with an optical path of $96 \mathrm{~m}$ and a resolution of $1 \mathrm{~cm}^{-1}$. The major product is formaldehyde, clearly identified by its main signatures at $1746,2765,2801$ and $2897 \mathrm{~cm}^{-1}$. Its formation as well as that of formic acid has been followed during the whole irradiation time, as shown in Fig. $4 a$ and $4 b$. From these results one can easily derive the production rate of formaldehyde from POM at $147 \mathrm{~nm}$. As shown in Fig. 5, it is quite constant as a function of time. The Xenon Lamp flux has been found to be of about $10^{16}$ quanta $\cdot \mathrm{s}^{-1}$ by chemical actinometry using $\mathrm{N}_{2} \mathrm{O}$ (Greiner, 1967). We found a value near unity for the quantum yield of formaldehyde production. We are currently studying the influence of the irradiation wavelength on this parameter. Then, we will be able to conclude if POM is the good candidate as parent molecule for the formaldehyde extended source.

\section{Conclusion}

Laboratory experiments are very useful in regard to theoretical models describing a planetary atmosphere or a cometary environment.

First, they provide the necessary fundamental parameters necessary to build up these models. A lot of experimental works are performed all over the world, but unfortunately, they are generally not carried out in the frame of planetary or cometary studies and hence are not representative of the pressure and temperature conditions of these environments. Furthermore, the error bars in the experimental values obtained are often large and their determination thus needs to be carried out with a better accuracy. In the case of the chemistry of comets, kinetic and photochemical data concerning the photodegradation of large molecules suspected to be present in the nucleus of comets are totally unknown. As a matter of fact, the knowledge of such data is crucial in order to elaborate theoretical models that can investigate the connection between the solid and gaseous phase of comets. In our laboratory we are currently developing an experimental program which allows us to obtain the quantum yields of formation of photodegradation products of high molecular weight compounds. The first application of this experimental program is the study of the photodegradation of polyoxymethylene, a polymer presented as the best candidate as parent molecule of formaldehyde extended source in the coma of comets.

Laboratory experiments can also be performed to simulate the atmosphere of a planet or the cometary environment. The direct comparison of the results obtained from these simulation experiments with those obtained from theoretical photochemical models, in our point of view, can provide useful information about the studied object. However, it 


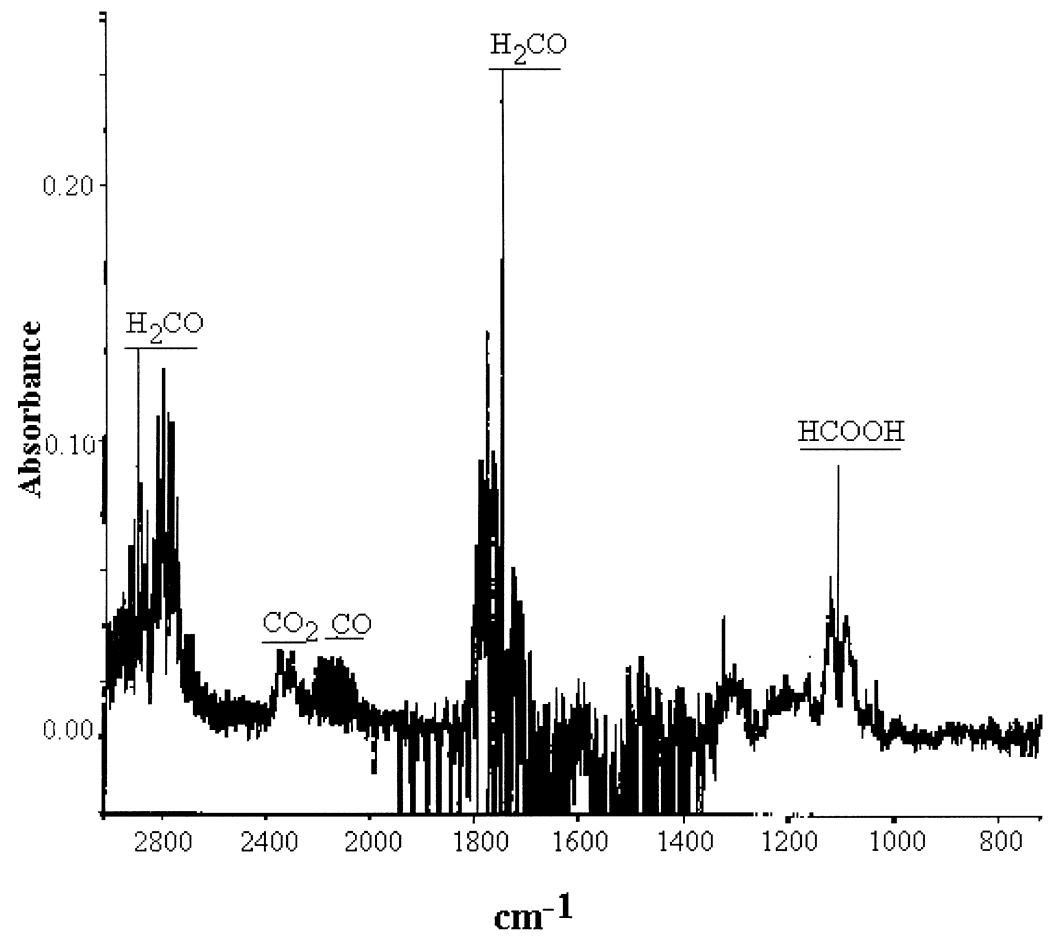

Fig. 3. Infrared spectrum recorded by the Atmospheric Simulation Chamber after a 4-h irradiation of POM. Optical path length: 96 m. Resolution: $1 \mathrm{~cm}^{-1}$.

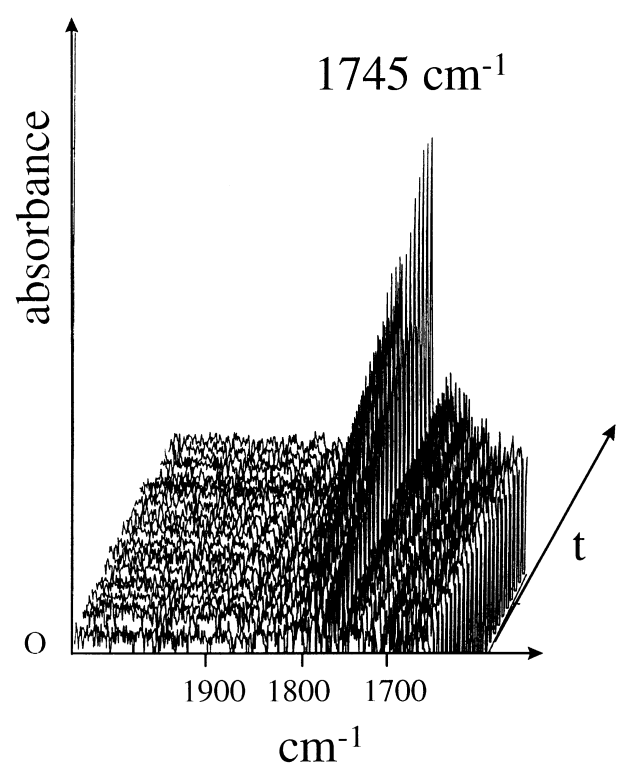

$4 \mathrm{a}: \mathrm{H}_{2} \mathrm{CO}$

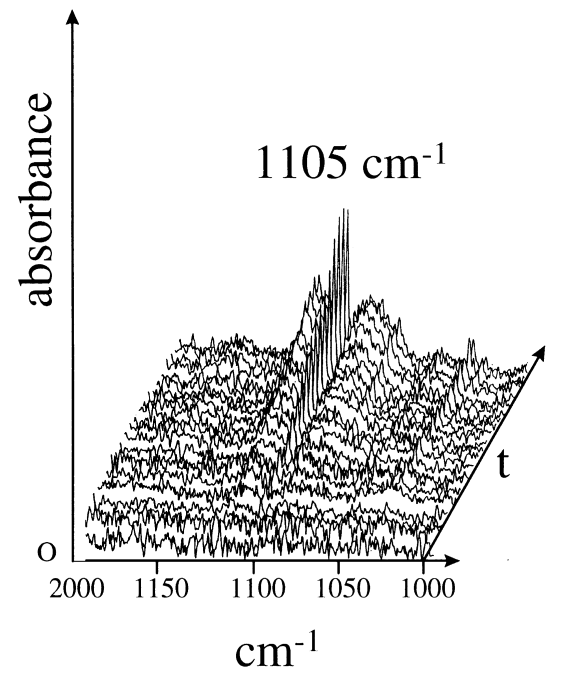

4b : $\mathrm{HCOOH}$

Fig. 4. (a and b) Evolution of absorbance of $\mathrm{H}_{2} \mathrm{CO}\left(1745 \mathrm{~cm}^{-1}\right)$ and $\mathrm{HCOOH}\left(1105 \mathrm{~cm}^{-1}\right)$ as a function of time. Time is represented here in arbitrary units. 


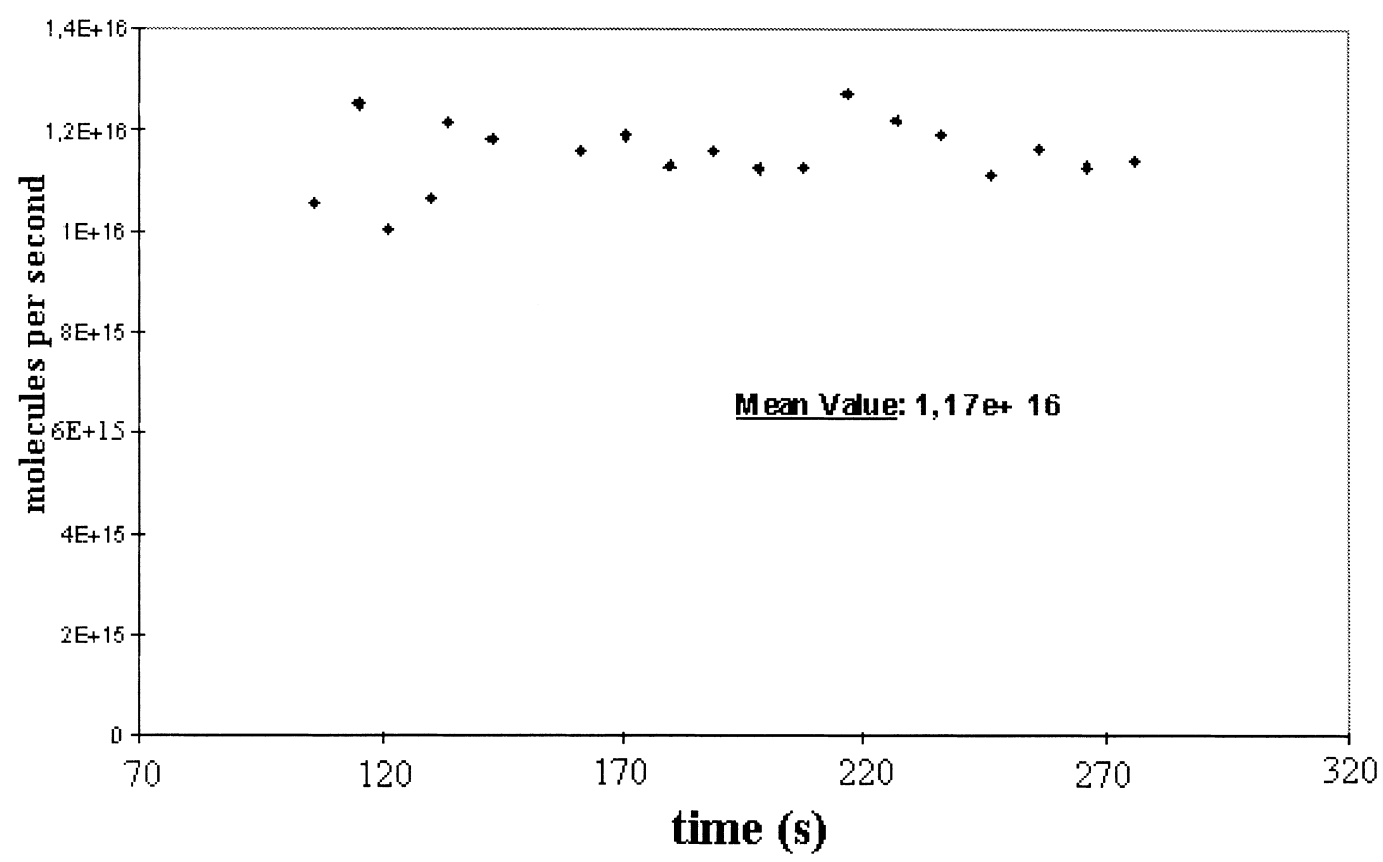

Fig. 5. Production rate of $\mathrm{H}_{2} \mathrm{CO}$ as a function of time.

turns out that the simulation experiments, as well as the chemical scheme used in the models, have strong limitations. However, such comparison is the only way to test the accuracy of the description of the mechanisms in the models. In order to go further in this program of coupling experiment and theory, we propose to reduce the complexity of the problem by carrying out laboratory experiments that mimic part of the chemical scheme of theoretical models. The first application of this kind of experiment is the test of the catalytic dissociation mechanism of methane via the photolysis of acetylene in Titan's stratosphere. The experimental conditions have been carefully investigated in order to minimize the experimental wall effects. The use of an atmospheric simulation chamber is the way to avoid such effects. Under these conditions, the data obtained can be constructively compared with a 0-D theoretical model. Improvement of the latter can be reached with iterations between theory and experiment.

In situ exploration of Titan and of cometary nuclei through space missions will take place in the near future, reinforcing the importance of laboratory experiments. The Cassini-Huygens mission, launched in October 1997, will release the Huygens probe into Titan's atmosphere in the autumn of 2004. The planned launch date for the NASA/ESA Rosetta mission is 2003. All data inferred from laboratory experiments as those described here will have direct implications in the analysis and interpretation of the data from these missions.

\section{References}

Bernstein, M.P., Sandford, S.A., Allamandola, L.J., Chang, S., Scharberg, M.A., 1995. Organic compounds produced by photolysis of realistic interstellar and cometary ice analogs containing methanol. Astrophys. J. 454, 327-344.

Bézard, B., Marten, A., Paubert, G., 1993. Detection of acetonitrile on Titan. Amer. Astron. Soc. 25, 1100.

Briggs, R., Ertem, G., Ferris, J.P., Greenberg, J.M., McCain, P.J., Mendoza-Gomez, C.X., Schutte, W., 1992. Comet Halley as an aggregate of interstellar dust and further evidence for the photochemical formation of organics in the interstellar medium. Origins Life Evol. Biosphere 22, 287-307.

Bruston, P., Raulin, F., 1995. Planetology and the origin of life: from space exploration to exobiology, and related interdiciplinary program. Planet. and Space Sci. 43, 1-2.

Coll, P., Coscia, D., Gazeau, M.-C., Guez, L., Raulin, F., 1998. Review and latest results of laboratory investigations of Titan's aerosols. Origins Life Evol. Biosphere 28, 195-213.

Coll, P., Coscia, D., Smith, N., Gazeau, M.-C., Ramirez, S.I., Cernogora, G. et al., 1999. Experimental laboratory simulation of Titan's atmosphere (aerosol and gas phase). Planet. and Space Sci. 47, 1331-1340.

Cottin, H., Gazeau, M.-C., Doussin, J.F., Raulin, F., 1999. S.E.M.A.Ph.Or.E COMETAIRE, a tool for the study of the photochemical decomposition of probable cometary large organic molecules. First application: Polyoxymethylene. Physic and Chemistry of the Earth 24, 597-604.

Cousténis, A., Salama, A., Encrenaz, T., Lellouch, E., Gautier, D., Kessler, M.F., et al., 1997. Titan's observation with ISO. Bull. Amer. Astron. Soc. DPS Meeting 29, 33.03.

Crifo, J.-F., Rodionov, A.-V., Bockelée-Morvan, D., 1999. The 
dependence of the circumnuclear coma structure on the properties of the nucleus III. First modeling of a CO-dominated coma, with application to Comets $46 \mathrm{P} /$ Wirtanen and $29 \mathrm{P} / \mathrm{Sch}$ assmannWachmann. Icarus 138, 85-106.

Crovisier, J., 1998. Physics and chemistry of comets: recent results from comets Hyakutake and Hale-Bopp. Faraday Discuss. 109, 437-457.

Daugherty, T.L., Davis, A.G., Phillips, W.J., Lay, L.T., 1995. Infrared Spectral Database on the World Wide Web in Optical Sensing for Environmental and Process Monitoring. Association, AWM, Virginia.

Daugherty, T.L., Phillips, W.J., Brown, D.G., Lay, L.T., 1994. High temperature database and spectral database. In: Optical Sensing for Environmental and Process Monitoring. Association, AWM, Virginia.

Doussin, J.F., Ritz, D., Jolibois, R.D., Monod, A., Carlier, P., 1997. Design of an environmental chamber for the study of atmospheric chemistry. Analysis 7, 236-242.

Gerakines, P.A., Schutte, W.A., Ehrenfreund, P., 1996. Ultraviolet processing of interstellar ice analogs. I. Pure ices. Astro. Astrophys. 312, 289-305.

Greiner, N.R., 1967. Photochemistry of $\mathrm{N}_{2} \mathrm{O}$ essential to a simplified vacuum-ultraviolet actinometer. J. Chem. Phys. 47 (11), 43734377.

Hidayat, T., Marten, A., Bézard, B., Gautier, D., Owen, T., Mattews, H.E., Paubert, G., 1997. Millimeter and submillimeter heterodyne observations of Titan, retrieval of the vertical profile of $\mathrm{HCN}$ and the C-12/C-13 ratio. Icarus 126, 170-182.

Huebner, W.F., 1987. First polymer in space identified in comet Halley. Science 237, 628-630.

Irvine, W.M., Bergin, E.A., Dickens, J.E., Jewitt, D., Lovell, A.J., Matthews, H.E., et al., 1998. Chemical processing in the coma as the source of cometary HNC. Nature 393, 547-550.

Kobayashi, K., Kasamatsu, T., Kaneko, T., Koike, J., Oshima, T., Saito, T., et al., 1995. Formation of amino acid precursors in cometary ice environments by cosmic radiation. Adv. in Space Res. 16, (221-(2)26.

Kuiper, G.P., 1944. Titan: a satellite with an atmosphere. Astrophys. J 100, 378.

Lara, L.M., Lellouch, E., López-Moreno, J.J., Rodrigo, R., 1996. Vertical distribution of Titan's atmospheric neutral constituents. J. Geophys. Res. 101 (E10), 23,261-23,283.

McDonald, G.D., Thompson, W.R., Heinrich, M., Khare, B., Sagan, C., 1994. Chemical investigation of Titan and Triton tholins. Icarus 108, 137-145.

Meier, R., Eberhardt, P., Krankowsky, D., Hodges, R.R., 1993. The extended formaldehyde source in comet $\mathrm{P} /$ Halley. Astron. Astrophys. 277, 677-691.

Mitchell, D.L., Lin, R.P., Carlson, C.W., Korth, A., Rème, H., Mendis, D.A., 1992. The origin of complex organic ions in the coma of comet Halley. Icarus 98, 125-133.
Möller, G., Jackson, W.M., 1990. Laboratory studies of polyoxymethylene: application to comets. Icarus 86, 189-197.

Moore, M.R., Hudson, R.L., 1998. Infrared study of ion-irradiated water-ice mixtures with hydrocarbons relevant to comets. Icarus $135,518-527$.

Notesco, G., Bar-Nun, A., 1996. Enrichment of CO over $\mathrm{N}_{2}$ by their trapping in amorphous ice and implication to comet $\mathrm{P} /$ Halley. Icarus $122,118-121$.

Notesco, G., Bar-Nun, A., 1997. Trapping of methanol, hydrogen cyanide, and $n$-hexane in water ice, above its transformation temperature to the crystalline form. Icarus 126, 336-341.

Notesco, G., Laufer, D., Bar-Nun, A., 1997. The source of the high $\mathrm{C}_{2} \mathrm{H}_{6} / \mathrm{CH}_{4}$ ratio in comet Hyakutake. Icarus 125, 471-473.

Raulin, F., Aflalaye, A., Bruston, P., Coll, P., Gazeau, M.-C., Sternberg, R., et al., 1996. In situ exploration of Titan and cometary nucleus: implication for exobiology. In: Chela-Flores, J., Raulin, F. (Eds.), Chemical Evolution: Physics of the Origin and Evolution of Life, pp. 357-371.

Rodgers, S.D., Charnley, S.B., 1998. HNC and HCN in Comets. Astrophys. J. Lett. 501 (2), L227-L230.

Sagan, C., Khare, B.N., Lewis, J.S., 1984. Organic Matter in the Saturn System. In: Gehrels, T., Matthews, M.S. (Eds.), Saturn. University of Arizona Press, Tucson, pp. 788-807.

Solà, J.C., 1908. Astron. Nach 179, 289.

Schutte, W.A., Allamandola, L.J., Sandford, S.A., 1993. An experimental study of the organic molecules produced in cometary and interstellar ice analogs by thermal formaldehyde reactions. Icarus 104, 118-137.

Smith, N.S., 1999. Sensibilité des modèles théoriques de l'atmosphère de Titan aux incertitudes sur la photochimie des hydrocarbures simples. Thèse de doctorat de l'Université Paris 12-Val de Marne.

Smith, N.S., Gazeau, M.-C., Khelifi, A., Raulin, F., 1999. A combined experimental and theoretical study of the catalytic dissociation of methane by the photolysis of acetylene at $185 \mathrm{~nm}$. Planet and Space Sci. 47, 3-10.

Strazzulla, G., Leto, G., Baratta, G.A., Spinella, F., 1991. Ion irradiation experiment relevant to cometary physics. J. Geophys. Res. 96 (E2), 17547-17552.

Strobel, D.F., 1974. The photochemistry of hydrocarbons in the atmosphere of Titan. Icarus 21, 466-470.

Thompson, W., Todd, H., Schwartz, J., Khare, B., Sagan, C., 1991. Plasma discharge in $\mathrm{N}_{2}+\mathrm{CH}_{4}$ at low pressure: experimental results and application to Titan. Icarus 90, 57-73.

Toublanc, D., Parisot, J.P., Brillet, J., Gautier, D., Raulin, F., McKay, C.P., 1995. Photochemical modelling of Titan's atmosphere. Icarus 113, 2-26.

De Vanssay, E., Gazeau, M.-C., Guillemin, J.-C., Raulin, F., 1995. Experimental simulation of Titan's organic chemistry at low temperature. Planet and Space Sci. 43, 25-31.

Yung, Y.L., Allen, M., Pinto, J.P., 1984. Photochemistry of the atmosphere of Titan: comparison between model and observation. Astrop. J. Supp. Ser. 55 (3), 465-506. 